EDITORIAL

\title{
Prediction of disease progression in idiopathic pulmonary fibrosis
}

\author{
A.U. Wells*, R.M. du Bois**
}

In idiopathic pulmonary fibrosis (lone cryptogenic fibrosing alveolitis (CFA)), there has been no change in survival or the frequency of responsiveness to therapy over the last two decades. Approximately $50 \%$ of patients die within 5 yrs of the onset of dyspnoea, and objective evidence of a response to therapy is seen in only $20 \%$ of cases [1-3]. These outcome statistics have engendered a certain nihilism about the management of CFA. In a survey of three regions in the UK, only two thirds of CFA patients are treated at all, and a minority undergo any form of biopsy procedure or bronchoalveolar lavage [4]. It is most unlikely that UK practice differs greatly from respiratory medical practice throughout Europe. This approach can be summarized as one of "primum non nocere": many practitioners are unwilling to utilize invasive diagnostic procedures and therapies notorious for their side-effects, without evidence that an aggressive approach is likely to be beneficial. As a result, many patients receive little intensive therapy early in the disease and obtain little benefit from intensive therapy when the disease is advanced.

Something needs to be done about this situation. In CFA, inflammation is widely believed to precede and lead to fibrosis; inflammatory disease is generally responsive to therapy. However, if early inflammatory disease is to be reversed, inflammatory disease must be treated early. If disease progression is to be prevented, progressive disease must be identified. It appears likely that thin section computed tomography (CT) will play a central role, both in securing an earlier diagnosis of CFA $[5,6]$ and in predicting responsiveness to treatment. In diffuse lung disease, a prominent ground-glass pattern on $\mathrm{CT}$ is indicative of potential reversibility [7]. In CFA, this pattern identifies an increased likelihood of inflammatory histology at open lung biopsy [8], and an improved outcome, independent of disease severity [9]; with the important caveat that ground-glass attenuation is less likely to regress when admixed with an equally extensive reticular pattern [10].

Unfortunately, CT is not the whole answer, for whilst it is able to identify reversible disease, it has yet to discriminate between stable and progressive fibrosing alveolitis. Most CFA patients have an irreversible,

\footnotetext{
*Royal Brompton National Heart and Lung Hospital, London, UK. **Department of Respiratory Medicine, Green Lane Hospital, Auckland, New Zealand. Correspondence: R.M. du Bois, Royal Brompton National Heart and Lung Hospital, Sydney St, London SW3 6NP, UK.
}

predominantly reticular pattern on $\mathrm{CT}$; because a reticular pattern represents established fibrosis and injury, a significant improvement with treatment is often unattainable, and the only realistic therapeutic goal is an exercise in damage-limitation - the prevention of further deterioration. However, the rate of progression in CFA is highly variable. The practitioner needs markers of progressive disease; a means of identifying patients at higher risk of decline would give clinicians the confidence to reserve potentially toxic treatments for those in need of them.

At the present time, this need has not been met. A bronchoalveolar lavage neutrophilia and/or eosinophilia has been advocated by some as a marker of progressive disease [11], but is insufficiently consistent to be applied with confidence in routine clinical practice. In a number of studies of outcome, a wide variety of functional indices have been predictive of mortality, including lung volumes, arterial gases and measures of gas exchange $[1,3,12]$. However, the derivation of markers of disease progression from survival data suffers from one important drawback; mortality is heavily influenced by the baseline severity of disease as well as by the rate of progression. In other words, functional indices associated with a poor survival may be indicative merely of advanced disease and not of the likely rate of decline in lung function per unit time.

The approach taken by AGusti et al. [13] offers new insights into the use of functional indices to identify patients at a higher risk of decline. In an elegant analysis of change in disease severity in CFA over a $3 \mathrm{yr}$ period, the authors were able to demonstrate that initial measurements of the carbon monoxide diffusing capacity and oxygen desaturation on exercise were both predictive of disease progression; whereas, a large number of other functional and clinical indices were not. The poor predictive value of such parameters as a crackle index and gallium uptake is perhaps not surprising, as their reproducibility is open to question. However, the same cannot be said for functional indices, such as spirometric lung volumes and arterial gases, which are used by most physicians in the routine evaluation of CFA. Agusti et al. [13] have advanced a compelling case for the routine use of the diffusing capacity and/or exercise testing in initial evaluation. If they are correct, less sophisticated but more easily measured functional indices are unequal to the task of identifying progressive disease.

The question of whether exercise testing and the measurement of diffusing capacity should both be routinely 
performed is rather more contentious. Exercise testing in CFA has its devotees, among them the authors of the present study, who have published previously in this area. In one often quoted American study [14], data from exercise testing was shown to correlate better with the severity of morphological findings at open lung biopsy than a wide range of other functions indices. However, Agusti et al. [13] have not, in fact, demonstrated that the use of exercise testing offers significant advantages over the measurement of diffusing capacity; after all, unlike exercise testing, the latter can be measured in almost all patients and now has a clinically acceptable reproducibility [15].

Another finding reported by Agusti et al. [13] merits particular emphasis; in their patients, arterial gases at rest did not change significantly during a 3 yr period, despite striking alterations in other functional indices. The detection of change in disease severity in CFA is often difficult; the CRP score, a composite clinical/ radiological/physiological index promulgated during the late 1980's, has been advocated for this purpose [16]. If the observations in the present study are confirmed, and declines in arterial gases at rest are found to occur only when disease is advanced, the inclusion of these indices in the CRP score in the evaluation of less advanced disease can be questioned.

The work of Agusti et al. [13] needs to be repeated. Although theirs is one of the larger recent series, the number of patients is small and many were lost to followup. The problem is compounded by the need to perform multiple statistical analyses; statistically significant findings in this population must, therefore, be interpreted with caution. AgUsti et al. [13] counter this criticism by arguing that their findings are not meaningless from a physiological viewpoint. They may be right: only time will tell.

Another potential marker of disease progression is the clearance of inhaled $99 \mathrm{~m}$ Tc-diethylenetriamine pentacetate (DTPA). In a recent retrospective study, abnormally rapid clearance of $99 \mathrm{~m}$ Tc-DTPA at initial measurement was found to identify fibrosing alveolitis patients at higher risk of a decline in functional indices over the subsequent 3 yrs [17]. LABRUNE et al. [18] set out to evaluate possible mechanisms of increased $99 \mathrm{~m}$ Tc-DTPA clearance in fibrosing alveolitis and, by studying the effects of corticosteroid therapy, were able to demonstrate a reversible and irreversible component of rapid clearance. The observation of decrease in clearance in response to treatment is important and further emphasizes the value of clearance measurements in determining the need for treatment, particularly as the greatest rates of clearance were associated with the largest improvement in vital capacity in response to treatment.

The authors conclude that rapid clearance is likely to reflect both interstitial inflammation and mechanical stretch of the respiratory epithelium due to lung fibrosis. This question is not straightforward; it has been argued that the intensity of inflammatory cell infiltration in the lung interstitium might, itself, be linked to the severity of lung fibrosis. As the authors point out, incomplete control of inflammation in the setting of significant lung fibrosis is an alternative explanation for fixed abnormalities in ${ }^{99 \mathrm{~m} T c-D T P A}$ clearance.

The pathogenic significance of abnormally rapid ${ }^{99 \mathrm{mTc}-}$ DTPA clearance is unclear. It is possible that increased clearance is merely a marker of ongoing inflammation and has no direct causative role in disease progression. However, an intriguing alternative hypothesis can be advanced on teleological grounds; limitations to epithelial permeability would not be tightly controlled in healthy individuals unless increases in clearance are potentially deleterious. The lung is constantly exposed to the environment; the increased penetration of a wide variety of inhaled antigens into the lung interstitium might play an important part in the acceleration of immunopathogenetic events. It is even possible that increased clearance from smoking might allow inhaled antigen to initiate disease. Whilst there is no compelling evidence for an inhaled antigen hypothesis, limited support is provided by a recent study in which an increased prevalence of CFA was found in individuals exposed to wood fires and metal dust [19].

Measures of gas exchange and epithelial permeability have the potential to play an important role in defining patients at risk of decline and justifying early therapeutic intervention. However, a number of important questions remained unanswered. The prognostic utility of combining these indices, together with findings on CT and, arguably, bronchoalveolar lavage, requires evaluation. Furthermore, it is unclear whether disease progression identified in these ways can be prevented by the earlier institution of treatment. The efficiency of steroid and/or immunosuppresive therapy has traditionally been measured by the number of patients whose disease has been improved. However, therapy may also be successful if it slows the rate of progression of disease in CFA, and this has never been properly studied. These evaluative studies need to be performed (possibly placebo-controlled?). Until this question has been addressed, the therapeutic value of identifying patients at higher risk will remain less certain, especially if arresting disease progression continues to be regarded as synonomous with failed treatment. A particularly important question to be answered is whether disease, once it has reached a certain stage, will progress inexorably, irrespective of therapy; the findings of AGUSTI et al. [13] may support this hypothesis. If this is the case, further weight is added to the argument for earlier treatment.

Patients with diffuse lung disease in general, but particularly CFA, have diseases for which there are no clear guidelines on quality of management. There is a reluctance to investigate and treat early because of the potential for side-effects of the investigations and drugs. Confidence to change these management strategies is required. The studies by AGUSTI et al. [13] and LABRUNE et al. [18] in this month's issue of the Journal, taken together with other recently published articles on prognostic indicators, have shown that it is now possible to design appropriate studies to answer these important issues in early and advanced disease, and in treated and untreated patients. The important question, notably the difficult problem of prevention of deterioration, will require studies involving 
reasonably large numbers of patients, seen in specialist centres with an interest in diffuse lung disorders and a multinational, co-ordinated strategy. For too long, these issues have been ignored. A much more logical approach is needed; we now have the opportunity to meet this challenge.

\section{References}

1. Stack BHR, Choo-Kang YFJ, Heard BE. The prognosis of cryptogenic fibrosing alveolitis. Thorax 1972; 27: 535-542.

2. Carrington CB, Gaensler EA, Coutu RE, FitzGerald MX, Gupta RG. Natural history and treated course of usual and desquamative interstitial pneumonia. $N$ Engl J Med 1978; 298: 801-809.

3. Turner-Warwick M, Burrows B, Johnson A. Cryptogenic fibrosing alveolitis: clinical features and their influence on survival. Thorax 1980; 35: 171-180.

4. Johnston IDA, Gomm SA, Kalra S, Woodcock AA, Evans $\mathrm{CC}$, Hind CRK. The management of cryptogenic fibrosing alveolitis in three regions of the United Kingdom. Eur Respir J 1993; 6: 891-893.

5. Mathieson JR, Mayo JR, Staples CA, Muller NL. Chronic diffuse infiltrative lung disease: comparison of diagnostic accuracy of CT and chest radiography. Radiology 1989; 171: 111-116.

6. Tung KT, Wells AU, Rubens MB, Kirk JME, du Bois RM, Hansell DM. Accuracy of typical computed tomographic appearances of fibrosing alveolitis. Thorax 1993; 48: 334-338.

7. Leung AN, Miller RR, Muller NL. Parenchymal opacification in chronic infiltrative lung diseases: CTpathologic correlation. Radiology 1993; 188: 209-214.

8. Muller NL, Staples CA, Miller RR, Vedal S, Thurlbeck WM, Ostrow DN. Disease activity in idiopathic pulmonary fibrosis: CT and pathologic correlation. Radiology 1987; 165: 731-734.

9. Wells AU, Hansell DM, Rubens MB, Cullinan P, Black
$\mathrm{CM}, \mathrm{Du}$ Bois RM. The predictive value of appearances on thin section computed tomography in fibrosing alveolitis. Am Rev Respir Dis 1993; 148: 1076-1082.

10. Wells AU, Rubens MB, du Bois RM, Hansell DM. Serial $\mathrm{CT}$ in fibrosing alveolitis: prognostic significance of the initial pattern. AJR 1993; 161: 1159-1165.

11. Watters LC, Schwarz MI, Cherniack RM. Idiopathic pulmonary fibrosis: pretreatment bronchoalveolar lavage cellular constituents and their relationships with lung histopathology and clinical response to therapy. Am Rev Respir Dis 1987; 135: 696-704.

12. Tukiainen P, Taskinen E, Holsti P, Korhola O, Valle M. Prognosis of cryptogenic fibrosing alveolitis. Thorax 1983; 38: 349-355.

13. Agusti C, Xaubet A, Agusti AGN, Roca J, Ramirez J, Rodriguez-Roisin R. Clinical and functional assessment of patients with idiopathic pulmonary fibrosis: results of a 3 year follow-up. Eur Respir J 1994; 7: 643-650.

14. Fulmer JD, Roberts WC, Von Gal ER, Crystal RG. Morphologic-physiologic correlates of the severity of fibrosis and degree of cellularity in idiopathic pulmonary fibrosis. J Clin Invest 1979; 63: 655-676.

15. Standardized lung function testing: official statement of the European Respiratory Society. Eur Respir J 1993; 6 (Suppl. 16): 1-100.

16. Watters LC, King TE, Schwarz MI, Waldron JA, Stanford RE, Cherniack RM. A clinical, radiographic and physiologic scoring system for the longitudinal assessment of patients with idiopathic pulmonary fibrosis. Am Rev Respir Dis 1986; 133: 97-103.

17. Wells AU, Hansell DM, Harrison NK, Lawrence R, Black CM, du Bois RM. Clearance of inhaled 99m-Tc-DTPA predicts the clinical course of fibrosing alveolitis. Eur Respir J 1993; 6: 797-802.

18. Labrune S, Chinet T, Collignon MA, Barritault L, Huchon GJ. Mechanisms of increased epithelial lung clearance of DTPA in diffuse fibrosing alveolitis. Eur Respir $J$ 1994; 7: 651-656.

19. Scott J, Johnston I, Britton J. What causes cryptogenic fibrosing alveolitis? A case-control study of environmental exposure to dust. Br Med J 1990; 301: 1015-1017. 